independent observers were then responsible for observing and recording times at which patients entered and exited the procedure room coupled with noting any issues for delays.

Results A total of 509 day case patients and 27 inpatient procedures were performed in 4 procedure rooms during the study period over 138 endoscopy lists. The adjusted median delay in starting time was 26 minutes with a peak of 20 minutes. The adjusted median turnaround times (time period between exit of patient from endoscopy room to entry of next patient in to the room) was $13: 11$ minutes with a peak at 12:30 minutes. The median for overruns (overrunning of lists beyond schedule) was 49:00 minutes. The adjusted median procedure time was 25:00 minutes. 95\% of endoscopy lists started late and $80 \%$ of the lists finished late. Bottlenecks identified were (1) delays in booking, (2) delays in patient preparation including enema, (3) inpatient transportation from and to wards and (4) timely endoscope availability. These were addressed by changing patient arrival times, additional support workers to support admission and patient preparation, introduction of co-ordinator to monitor lists with empowerment to redeploy staffing, and the role of a 'runner' to liaise between decontamination and the procedure rooms.

Conclusion The 'time and motion' study is a useful tool to identify areas for improvement in patient flow and improve efficiency in the Endoscopy unit.

\section{PTU-082 DEVELOPMENT OF LOCAL ALCOHOL SERVICES - EXPERIENCE FROM AN ENGLISH RURAL DISTRICT GENERAL HOSPITAL}

Rosie Firth Burnside*, Evelyn Lim, Brian Ho, Piyush Singh, Brian Horgan, Albert H Davies, John Keating. University Hospitals of Morecambe Bay NHS Trust, Barrow-In-Furness, UK

\subsection{6/gutjnl-2019-BSGAbstracts.441}

Introduction Alcohol services are the cornerstone to delivering good alcohol detoxification treatment and outcomes for patients with alcohol dependence. The impact of staff educational program, e-CIWA proforma and inpatient outreach service for community rehabilitation (The Well) is assessed in this two-cycle audit study, leading to improvement in patient care and ongoing local service development at an English rural district general hospital.

Methods The 1st audit cycle was carried out between September 2016 and August 2017 at the Furness General Hospital in Cumbria. Adult patients admitted to Gastroenterology with alcohol related ICD-10 codings and an inpatient stay $>24$ hours were included in the study. A set proforma was used for data collection from the local electronic health record assessing standard of care against NICE recommendations. Outcomes assessed include patient engagement with inpatient and community services, discharge with detox medication and attainment of help in community. Quality deficiencies identified lead to introduction of hospital inpatient outreach service for community rehabilitation (The Well Community team) funded by substance misuse service Unity, electronic CIWA proforma and staff educational program in August 2017. A repeat audit cycle between September 2017 and August 2018 was conducted to assess impact of intervention. Chi-squared/ Fisher exact test was used for proportions testing with $\mathrm{p}<0.05$ considered as statistically significant.
Results Baseline demographics between 2016/17 audit $(n=73)$ and 2017/18 audit cycle $(n=86)$ are similar. Comparing the latter audit cycle to the form, there is an increase in Magnesium level testing (34.3 to $73.3 \%, \mathrm{p}<0.0001)$ and IV Pabrinex prescription ( 89 to $97.7 \%, \mathrm{p}<0.02$ ). However there is a reduction in CIWA assessment documentation $(56.4 \%$ to $43.6 \%, \mathrm{p}<0.0091)$ and oral Thiamine prescription $(97.3 \%$ to $76.7 \%, \mathrm{p}=0.0002)$ respectively. Attainment of help by patients after discharge in the community increased (11 to $38.4 \%$, $\mathrm{p}<0.0001)$. There is no change in frequency of motivational assessment, prescription of community detox medication on discharge or referral to community rehabilitation. However, total referrals received from all sources to Unity have increased by $20 \%$.

Conclusions Infrastructure development (The Well) and staff education leads to improvement in quality of alcohol detoxification care. Integration of inpatient community rehabilitation service remains a challenge in the rural setting and needs further promoting. Reduction in CIWA assessment suggests need for dedicated QI focus for e-CIWA use. Repeated audit cycles allow impact assessment of service development measure and further study on impact towards re-admission rates and cost benefit are needed.

\section{\begin{tabular}{|l|l}
\hline PTU-083 TIME OF DAY INFLUENCES COLONOSCOPY OUTCOME \\
\hline
\end{tabular} MEASURES}

Talia Caspi ${ }^{*}$, Harshil Patel, Pradeep Mundre. Bradford Royal Infirmary, Bradford, UK

\subsection{6/gutjnl-2019-BSGAbstracts.442}

Introduction Endoscopic surveillance and removal of adenomas significantly reduces the incidence and mortality of colorectal cancer. There is concern that time of day could affect colonoscopy outcomes due to endoscopist fatigue over the course of the day. This could lead to interval cancers, and ultimately increased mortality. However, there are few studies in this area, and they have yielded inconsistent results. The aim of this study was to investigate whether evening colonoscopy lists achieve the same outcomes as daytime lists.

Methods Colonoscopy data from patients on the symptomatic pathway was gathered retrospectively over a one year period. Data was gathered only from endoscopists doing both daytime and evening lists. Data collected included quality of bowel prep, caecal intubation rate, adenoma detection rate (ADR), and colorectal cancer detection. Colonoscopy outcome data was compared between daytime and evening lists. Data was analysed using the $\chi^{2}$ test, and Fishers exact test.

Results 1150 colonoscopies were included in the analysis, of which 845 were from daytime lists, and 305 were from evening lists. Time of day produced a near significant difference in ADR [23.2\% $(n=196)$ daytime vs $17.97 \%(n=55)$ evening, $\mathrm{p}=0.0581]$. Detection of colorectal cancer was significantly lower in evening lists $[2.13 \% \quad(\mathrm{n}=18)$ daytime vs $0.33 \%$ $(n=1)$ evening, $p<0.05]$. Caecal intubation rate was significantly lower during evening lists $[96.69 \%(n=817)$ daytime vs 94.43\% ( $\mathrm{n}=305)$ evening, $\mathrm{p}<0.05$ ].

Conclusions It has been shown that the time of day significantly affects some outcome measures of colonoscopies. Demand for colonoscopies in the UK is rising year on year, and evening lists help to meet this demand. However, it must 
be ensured that patient care is not compromised, and outcome measures remain stable over the course of the day.

\section{PTU-084 THE USE OF PANORAMIC CAPSULE ENDOSCOPY IN OBSCURE GASTROINTESTINAL BLEEDING}

Stefania Chetcuti Zammit*, Mark E McAlindon, Reena Sidhu. Sheffield Teaching Hospitals, Sheffield, UK

\subsection{6/gutjnl-2019-BSGAbstracts.443}

Introduction Up to $64 \%$ of patients with obscure gastrointestinal bleeding (OGIB) have lesions that can be reached with a standard gastroscope [1]. Positive findings outside the small bowel (SB) have been detected on axial small bowel capsule endoscopy (SBCE) (PillCam system, (Given Imaging) in patients with OGIB in up to $22 \%$ of patients in the stomach and $6 \%$ in the colon [2]. Our aim was to assess the diagnostic yield (DY) of panoramic SBCE (Capsocam) for the upper gastrointestinal tract, SB and colon in patients with OGIB.

Methods Patients at a tertiary centre who underwent Capsocam as part of the examination of OGIB following negative gastroduodenoscopy and colonoscopy or CT colonography were included. This study was carried out over a 6 year period. Findings on SBCE were recorded.

Results Forty-eight patients (37, 77.1\% overt, 11, 22.9\% occult OGIB) were included in this study. Thirty $(62.5 \%)$ were males. Mean age was $67.5 \pm 16.5$ years. OGIB was present for a mean of 19.9 SD \pm 92 months. Only one SBCE (9.1\%) was incomplete within the occult OGIB group $(p=0.229)$. The panoramic SBCE had a diagnostic yield of $37.5 \%$ (18 patients) in the SB. It also picked up additional findings in the stomach $(n=5,10.4 \%)$ and in the colon $(n=3$, $6.3 \%$ ). Some patients had more than one pathology (table 1).

\begin{tabular}{|c|c|c|c|}
\hline Findings & Stomach n (\%) & Small bowel n (\%) & Colon $\mathrm{n}(\%)$ \\
\hline Blood & $1(2.1)$ & $8(16.7)$ & $3(6.3)$ \\
\hline Erosions/gastritis & $3(6.3)$ & $3(6.3)$ & 0 \\
\hline Ulcers & $1(2.1)$ & $1(2.1)$ & 0 \\
\hline Angioectasias & 0 & $8(16.7)$ & $1(2.1 \%)$ \\
\hline ? tumour & 0 & $1(2.1)$ & 0 \\
\hline Diverticulum & 0 & $2(4.2)$ & 0 \\
\hline
\end{tabular}

Conclusions Panoramic SBCE can be useful in the detection of lesions in the stomach and colon missed during upper and lower gastrointestinal examinations. It can also be better in the detection of pathologies in overt OGIB than in occult OGIB.

\section{REFERENCES}

1. Zaman A, Katon RM: Push enteroscopy for obscure gastrointestinal bleeding yields a high incidence of proximal lesions within reach of a standard endoscope. Gastrointest Endosc 1998, 47:37-76.

2. Riccioni ME, Urgesi R, Cianci R, Marmo C, Galasso D, Costamagna G: Obscure recurrent gastrointestinal bleeding: a revealed mystery? Scand I Gastroenterol 2014, 49:102-026.

\section{PTU-085 VIRTUAL CLINICS OFFER AN EFFECTIVE AND SAFE ADDITION TO STANDARD OUT-PATIENT HEPATOLOGY SERVICES}

${ }^{1}$ Lynsey Corless*, ${ }^{2}$ Shaw Keith Lau, ${ }^{2}$ Alison Stainsby. ${ }^{1}$ Hull and East Yorkshire Hospitals, UK; ${ }^{2}$ Hull York Medical School, UK

\subsection{6/gutjnl-2019-BSGAbstracts.444}

Introduction Many patients with abnormal liver function tests/ steatosis do not have serious liver disease, and risk assessment for advanced disease can often be performed without face to face out-patient (OPC) review using laboratory results and other data. Virtual clinics (VC) may offer a useful adjunct to OPC increasing capacity for those requiring specialist care, and reduced patient visits. We piloted a consultant-led VC and assessed impact on OPC use.

Methods A retrospective audit was undertaken examining records of patients referred to liver VC/OPC (excluding viral hepatitis) in the year July 201-8. Clinical/demographic data were collected including referral detail, investigation results, and outcome of review. Appropriateness of referral route was reviewed by a consultant taking into account need for, and number of, OPC visits.

Results

VC 84 patients had initial review in VC. $72(85.7 \%)$ were community referrals, and $12(14.3 \%)$ from other specialties. There were no documented safety issues in patients managed in VC. 50 (59.5\%) were discharged after VC review, including $45(62.5 \%)$ of community referrals, equivalent to 4.2 unused OPC per month. Most were people with abnormal liver biochemistry or steatosis but low risk Fib4 score. Of 34 (40.5\%) who required subsequent OPC assessment 3 did not attend, $12(14.3 \%)$ required just 1 visit before discharge, $13(15.5 \%)$ needed - OPC, and $6(7.1 \%)$ entered long term follow up. In total $64.7 \%(22 / 34)$ of OPC need was solely for fibrosis assessment by Fibroscan, with 9/22 (40.9\%) discharged with normal result.

OPC OPC referrals were reviewed $(n=703)$, to determine how many would have been suitable for initial VC assessment. 456 first OPC attendances were identified after excluding cancellations/non-attenders. $40 \quad(8.7 \%)$ were discharged immediately, and $86(18.5 \%)$ were awaiting results to guide follow-up decision. These groups were considered potentially appropriate for VC, and reviewed in detail. Those placed on follow-up pathways were unlikely to be suitable and were not analysed further. Of those discharged at visit 1, 30 $(75 \%)$ would have been suitable for first review in VC, with 25 of those not requiring OPC attendance at all. Of 86 awaiting results, $58(67.4 \%)$ were suitable for first review in VC with 27 requiring no OPC input. A further 15 (17.4\%) could have avoided at least 1 visit. Taken together, this equates to $88 / 456(19.3 \%)$ visits which could have been conducted by VC, of whom 52/456 (11.4\%) required no OPC input at all. Including the 15 potentially avoidable visits, a total 67 OPC (5.6/month) could be saved $(9.5 \%$ of all OPC referrals).

Conclusions Most VC patients can be safely discharged without need for OPC, and expansion could further increase capacity for those requiring face-to-face specialist care. All our new referals are now initially vetted by VC. 